\title{
Synchrotron radiation complex ISI-800
}

\author{
V. Nemoshkalenko, V. Molodkin, A. Shpak, E. Bulyak*, I. Karnaukhov*, A. Shcherbakov* \\ and A. Zelinsky*
}

Institute of Metal Physics, Academy of Science of Ukraine, 252142 Kiev, Ukraine

* Kharkov Institute of Physics and Technology, 310108 Kharkov, Ukraine

\begin{abstract}
Basis considerations for the choice of a synchrotron light source dedicated for Ukrainian National Synchrotron Centre is presented. Considering experiments and technological processes to be carried out $800 \mathrm{MeV}$ compact four superperiod electron ring of third generation was chosen. The synchrotron light generated by the electron beam with current up to $200 \mathrm{~mA}$ and the radiation emittance of $2.7 * 10^{-8} \mathrm{~m} *$ rad would be utilized by 24 beam lines. Each line will provide the light beam with spectral brightness of $(2-9)^{*} 10^{20}$ phot $/\left(\mathrm{m}^{2 *} \mathrm{rad}^{2}\right)$ within $0.01 \%$ bandwidth. Two wigglers and an undulator will be inserted into the magnet lattice. The lattice is to provide large enough dynamic aperture and to decrease sensitivity to collective effects. The central magnet of each superperiod can be optionally replaced by a superconducting one to harden photon spectrum. The $120 \mathrm{MeV}$ traveling wave linac is intended to employ as an injector. The injector is placed below the ring to provide room for more beamlines. For this purpose the injecting system and the RF cavity share the same long straight section.
\end{abstract}

\section{INTRODUCTION}

The use of synchrotron radiation noted for its broad continuous and high brightness has brought the research in the fields of molecular physics, physics of condensed states of matter, chemistry, microbiology, etc. to a qualitatively higher level. In recent years, great interest has been displayed in basic synchrotron radiation sources whose physical properties along with performance parameters provide a means for handling process problems of X-ray lithography in microelectronics (photon energy $\varepsilon_{\gamma} \sim 1 \mathrm{keV}$ ), micromechanics $\left(\varepsilon_{\gamma} \sim 6 \mathrm{keV}\right)$ and angiography $\left(\varepsilon_{\gamma} \sim 33 \mathrm{keV}\right)$. The design of a relatively cheap and compact source with the above-mentioned photon beam parameters is a complicated task, since the compactness of the source sets limits, first of all, on the electron energy, and hence, on the energy of emitted photons. This, in turn, necessitates the mounting, arrangement of special devices such as superconducting wigglers with limiting magnetic-field values of about $10 \mathrm{~T}$. However, the installation of such wigglers in the storage rings with a beam energy lower than $1 \mathrm{Gev}$ is impeded by an impact of magnets on the beam focusing and phase stability of the orbit in the storage ring. This makes necessary long straight sections in the lattice to accommodate the matching elements, and also imposes special requirements on the wiggler design. Furthermore, in consequence of a great distortion of the reference orbit in the wiggler $(\Delta x \sim 5 \mathrm{~cm}$ at an electron energy of $0.8 \mathrm{GeV}$ and a field of $10 \mathrm{~T}$ in the wiggler), there arise essential technological difficulties. This paper describes the concept of a SR source which can form the basis of creating the National Centre of SR [1]. Ways of realizing this concept are discussed. 


\section{CHOICE OF THE SOURCE PARAMETERS}

The upper limit of the magnetic-field strength range, at which the magnetic materials can ensure a highquality field, is about $1.5 \mathrm{~T}$. Besides the radiation from bending magnets, the modern SR sources incorporate superconducting wigglers (with the field up to $10 \mathrm{~T}$ ) to increase the energy of the photons produced. The electron beam energy of about $800 \mathrm{MeV}$ allows the generation of photon beams complying with the requirements of most applications.

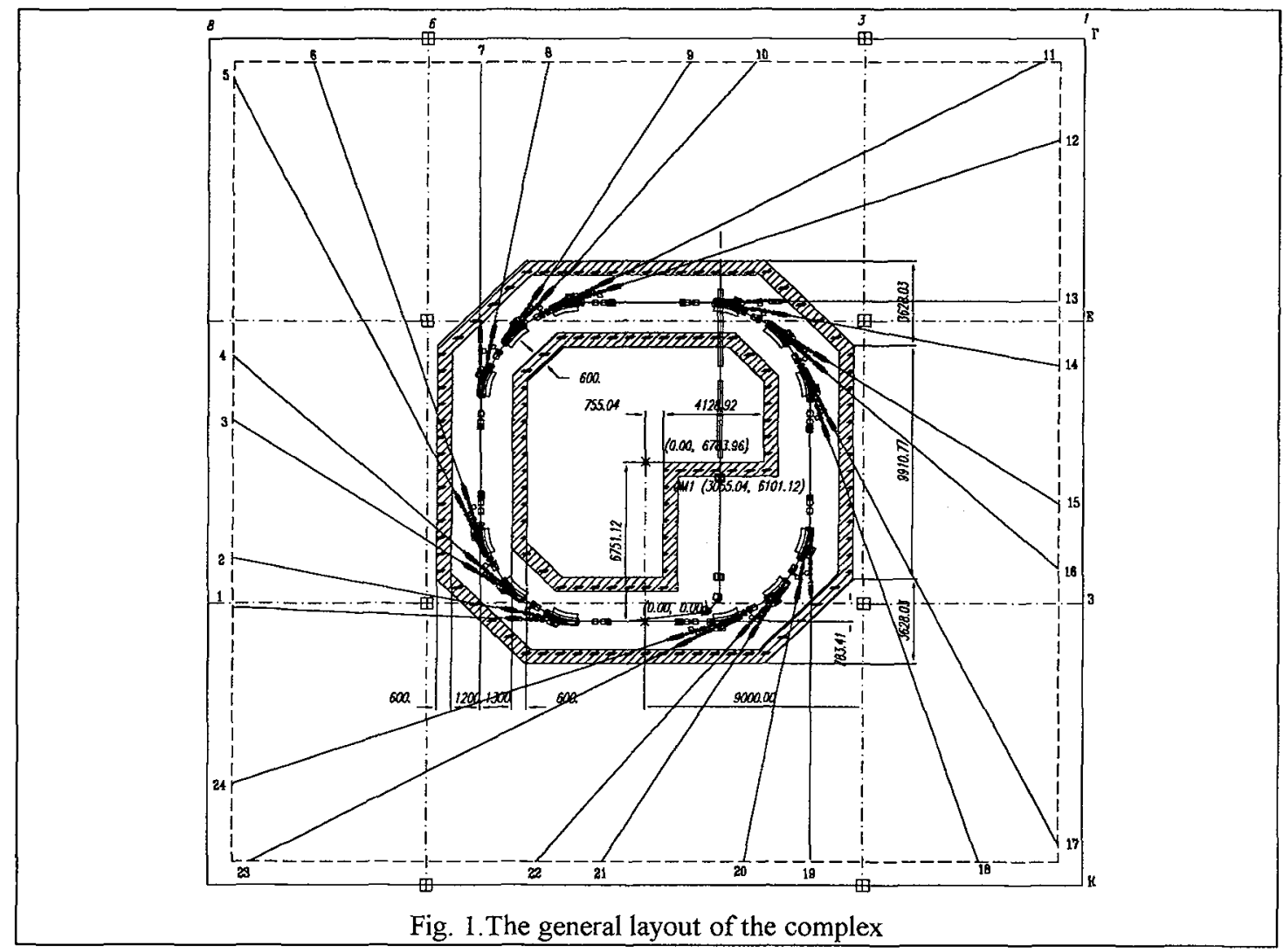

We came to conclusion that SR source would be a $800 \mathrm{MeV}$ compact electron storage ring. Injecting energy was chosen to be $120 \mathrm{MeV}$. The traveling wave linac is intended to employ as an injector. The injector is placed below the ring to provide room for more beamlines. For this purpose the injecting system and the RF cavity share the same long straight section. The general layout of the complex is presented in Fig. 1 .

The magnet lattice is responsible for both the efficient operation of the source and the SR beam quality. One of the principal SR beam parameters, i.e., brightness, is proportional to the transverse electron-beam density in the region of radiation. Therefore, it is necessary to have a beam with a minimal cross-sectional area; the lattice must ensure the minimal beam emittance and a low value of the vertical betatron function in bending magnets. The latter allows one to diminish the magnet gap, thereby reducing the cost of magnet manufacture and operation.

We have chosen the TBA lattice as providing low emittance. It consists of four superperiods. The structural formula of each superperiod has the form 


$$
\mathrm{O}_{1} \mathrm{QD} \mathrm{O}_{2} \mathrm{QF}_{1} \mathrm{O}_{3} \mathrm{QF}_{1} \mathrm{O}_{2} \mathrm{QD} \mathrm{O}_{1} \mathrm{MO}_{4} \mathrm{QF}_{2} \mathrm{O}_{4} \mathrm{MO}_{4} \mathrm{QF}_{2} \mathrm{O}_{4} \mathrm{M}
$$

where $\mathrm{QD}, \mathrm{QF}_{\mathrm{i}}$ are the horizontally defocusing and focusing quadrupoles; $\mathrm{M}$ is the dipole magnet, $\mathrm{O}_{\mathrm{i}}$ is the straight section; $\mathrm{O}_{1}=0.7 \mathrm{~m} ; \mathrm{O}_{2}=0.15 \mathrm{~m} ; \mathrm{O}_{3}=3.23 \mathrm{~m} ; \mathrm{O}_{4}=0.6 \mathrm{~m}$.

The curvilinear part of the trajectory includes: 3 magnets, each with a bending angle of $30^{\circ}$, the curvature radius $R=2.005 \mathrm{~m}(\mathrm{~B}=1.34 \mathrm{~T}$ at an electron energy $\mathrm{E}=0.8 \mathrm{GeV}, \mathrm{B}$ is the magnetic field strength at the equilibrium orbit), the field index $n=3$ and 2 focusing quadrupoles providing the achromaticity of the long straight part of the orbit.

The straight-line part of the trajectory comprises four quadrupole magnets which ensure the stability of radial and vertical motion in combination with two quadrupole magnets and vertically defocusing dipole magnets. Fig. 2 shows the machine functions of the superperiod for the zero dispersion function in the long straight section.

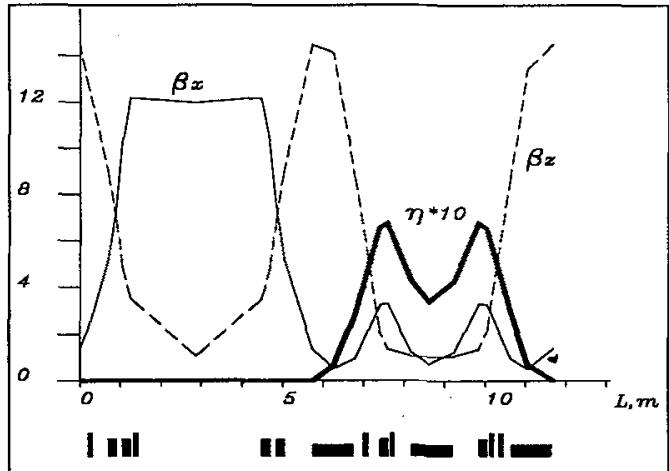

Fig. 2. The machine functions of a superperiod.

The phase advance for the focusing period is $\mu_{\mathrm{x}}=6.666$ and $\mu_{z}=5.026$ for radial and vertical oscillations, respectively. These values correspond to the tunes $Q_{x}=$ 4.25 and $Q_{z}=3.20$. Since the vertical focusing is mainly accomplished by gradient dipole magnets, this provides the smallest value of the betatron radial function $\beta_{x}$ in the magnet, and hence, the minimum radial size of the beam. The radiation emittance is $\varepsilon_{\mathrm{x}}=2.7 \cdot 10^{-8} \mathrm{mrad}(\mathrm{E}=0.8 \mathrm{GeV})$ for the steady-state energy straggling $\Delta p / p=5.88 \cdot 10^{-4}(E=0.8 \mathrm{GeV})$

The operating conditions of the SR source are characterized by a low radiation emittance attained due to a higher rigidity of the magnet lattice. This has resulted in the increased natural chromaticity, the compensation of which requires the mounting of sextupole lenses arranged in the sections with a nonzero dispersion. At large betatron amplitudes the presence of sextupole fields leads to the occurrence of undesirable nonlinear effects, which significantly decrease the dynamic aperture. The simulation has resulted in the following conclusions:

- the inclusion of sextupole lenses to correct the dynamic aperture appreciably increases the latter both in the absence and presence of sextupole perturbations in the dipole magnet;

- with the corrected equilibrium orbit and the compensation lenses switched on the dynamic aperture exceeds the geometric one, and therefore, will not affect the beam parameters.

The multiple injection chosen for this design consists in the following. The beam from the injector is horizontally introduced, via the transport system, to the vacuum chamber of the ring, where it is bent by the necessary angle with the help of the septum magnet. By the moment of injection of the next beam portion the orbit of the ring in the region of injection comes closer to the septum. After trapping the necessary number of turns, the orbit is restored, the initial betatron oscillations are damped.

\section{2,1. Superconducting wigglers}

The ISI-800 structure is anticipated to accommodate superconducting three-pole wigglers with the greatest field up to $10 \mathrm{~T}$ in the centre of long straight sections. A wiggler with such a field makes it possible to increase the critical photon beam energy up to $4.22 \mathrm{keV}$, thus enabling the performance of experiments in X-ray tomography, crystallography, EXAFS spectroscopy and also to generate photon beams with an intensity of about $\sim 10^{10} \mathrm{phot} / \mathrm{mrad}^{2} / \mathrm{s}$ (for X-ray holography applications). The incorpo- 
ration of such a device into the ring focusing structure without taking particular steps would result in an essential frequency shift and in the change of ring focusing functions.

Fig. 3 shows the arrangement of the insert consisting of a wiggler and the compensating structure. The insert enables one to obtain an arbitrary matrix of focusing by varying the strength of the quadrupole lenses. We have chosen the following concept of compensation for the wiggler effect: the focusing matrix of a long straight section remains unchanged irrespective of the operating conditions, while the values of the matrix elements of the wiggler-comprising section correspond to the same values of the matrix of the section without a wiggler, and this allows us to keep the focusing functions of the ring unchanged, except for the section inside the insert. Table 1 lists the values of insert parameters.

It should be noted that the restriction imposed on the strength of quadrupole lenses necessitates the change in the focusing properties of the central wiggler magnet.

Table 1. Parameters of insert

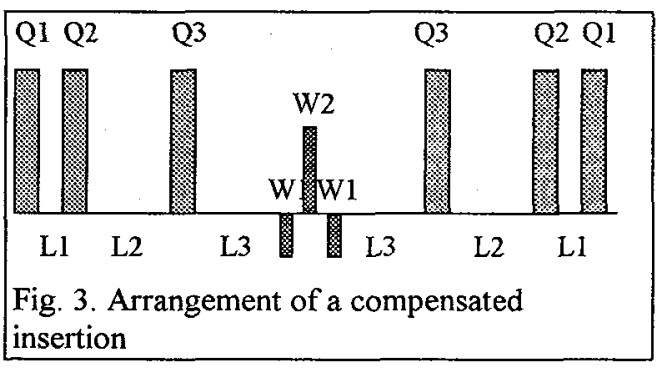

\begin{tabular}{lll}
\hline $\begin{array}{l}\text { Elemen } \\
\mathrm{t}\end{array}$ & Value & Length \\
\hline $\mathrm{Q} 1$ & $\mathrm{~K} 1=2.65 \mathrm{~T} / \mathrm{m}$ & $0.2 \mathrm{~m}$ \\
$\mathrm{Q} 2$ & $\mathrm{~K} 1=-11.95 \mathrm{~T} / \mathrm{m}$ & $0.2 \mathrm{~m}$ \\
$\mathrm{Q} 3$ & $\mathrm{~K} 1=13.16 \mathrm{~T} / \mathrm{m}$ & $0.2 \mathrm{~m}$ \\
$\mathrm{~W} 1$ & $\mathrm{H}=-5.0 \mathrm{~T}, \mathrm{ANG}=-12.88$ & $0.12 \mathrm{~m}$ \\
$\mathrm{~W} 2$ & $\mathrm{H}=10 \mathrm{~T}, \mathrm{ANG}=-5.44$ & $0.12 \mathrm{~m}$ \\
$\mathrm{~L} 1$ & & $0.15 \mathrm{~m}$ \\
$\mathrm{~L} 2$ & & $0.616 \mathrm{~m}$ \\
$\mathrm{~L} 3$ & & $0.5 \mathrm{~m}$ \\
\hline
\end{tabular}

The beam size at the radiation point is $\sigma_{x}=0.19 \mathrm{~mm}, \sigma_{z}=0.073 \mathrm{~mm}$. The $33 \mathrm{keV}$ SR brightness at an electron beam current of $0.2 \mathrm{~A}$ and an electron energy of $800 \mathrm{MeV}$ is $6.39^{*} 10^{9} \mathrm{~mm}^{2 *} \mathrm{mrad}^{*} 0.1 \% \lambda / \mathrm{s}$.

The calculations of the ISI-800 dynamic aperture on incorporating the wiggler have shown that its value is twice as that of the geometrical aperture in both transverse coordinates, and hence, it will not limit the parameters of the circulating beam.

The matched inserts can be installed in three long straight sections of the ring, thereby providing three channels of hard synchrotron radiation.

\subsection{A modified TBA lattice}

To produce high-brightness photon beams with a sufficiently high energy, we also considered a compact storage ring with the lattice based on the combined TBA cell ("warm" and superconducting magnets), where the central dipole magnet of the cell is superconducting.

The contribution of the central superconducting magnet (field is $10 \mathrm{~T}$ ) to the emittance value at a beam energy of $0.8 \mathrm{GeV}$ is $\varepsilon_{x}=2 * 10^{-8} \mathrm{mrad}$.

Fig. 4 shows the machine functions for the the modified TBA cell of the ISI- 800 storage ring. For comparison, Table 2 lists the values of the parameters for different variants of the ISI-800 storage ring lattice. 
Table 2 Parameters of the ISI-800 storage ring

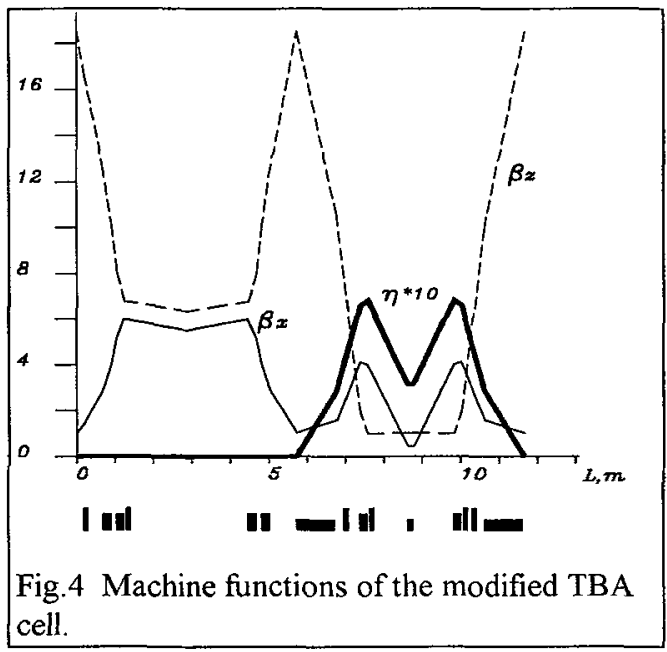

Fig. 4 Machine functions of the modified TBA
cell.

\begin{tabular}{lcc}
\hline \multicolumn{1}{c}{ Parameter } & TBA & Mod TBA \\
\hline Nominal energy, $\mathrm{MeV}$ & 800 & 800 \\
Stored current, $\mathrm{mA}$ & 200 & 200 \\
Circumference, $\mathrm{m}$ & 46.729 & 46.815 \\
Number of dipole magnets & 12 & $8+4$ \\
Radius of magnet bend, $\mathrm{m}$ & 2.005 & $2.005 / 0.27$ \\
Magnet length, $\mathrm{m}$ & 1.05 & $1.05 / 0.14$ \\
Betatron numbers, $v_{\mathrm{X}} / v_{\mathrm{Z}}$ & $4.26 / 3.20$ & $4.26 / 3.20$ \\
Compaction factor, $\alpha$ & 0.025 & 0.021 \\
Nat chromaticity, $\xi_{\mathrm{X}} / \xi_{\mathrm{Z}}$ & $-7.27 /-7.24$ & $-6.2 /-6.7$ \\
Damping times $\tau_{\mathrm{X}} / \tau_{\mathrm{z}} / \tau_{\mathrm{S}}$, & $8.7 / 13.8 / 9.8$ & $8.4 / 9.9 / 5.4$ \\
Emittance, mrad & $2.7 \cdot 10^{-8}$ & $4 . \cdot 10^{-8}$ \\
Beam size in centre of $\mathrm{SC}$ & & $0.15 / 0.07$ \\
Energy spread, $\%$ & 0.058 & 0.125 \\
Losses per turn, $\mathrm{keV}$ & 18.3 & 57.4 \\
Crit. energy $\mathrm{SC}, \mathrm{keV}$ & & 4.2 \\
\hline
\end{tabular}

The simulation of the dynamic aperture of the storage ring with a modified lattice shows that the presence of the superconducting central magnet in the TBA lattice exerts no essential influence on the aperture value.

Thus, from the comparison between the characteristics of the ISI- 800 based on traditional "warm" magnets with special inserts (of wiggler type) and the combined lattice with the central superconducting magnet in the TBA cell it is evident that the latter has the following advantages:

(i) a greater number of channels for photon beams with a hard spectrum;

(ii) a wide range of beam emittance variation with the storage ring tune remaining the same $\left(\varepsilon_{x}=4 \cdot 10^{-8} \div 6 \cdot 10^{-7} \mathrm{mrad}\right)$;

(iii) physically, the superconducting dipole magnet is considerably easier in manufacture than the wiggler is;

(iv) the influence of superconducting dipole magnets in the centre of the TBA cell on the beam dynamics and the actual aperture is insignificant because of the smallness of the structure functions at the place of magnet location.

The construction of the synchrotron radiation source ISI-800 with the combined TBA cell as a basis will ensure the conduction of photon beam studies in the ranges of vacuum ultraviolet and hard $\mathrm{X}$-ray radiation.

\subsection{SR beamlines}

The synchrotron light generated by the electron beam with current up to $200 \mathrm{~mA}$ and the radiation emittance of $2.7 \cdot 10^{-8} \mathrm{~m} \cdot \mathrm{rad}$ would be utilized by beam lines. Each line will provide the light beam with spectral brightness of (2-9) $10^{20}$ phot $/\left(\mathrm{m}^{2} \cdot \mathrm{rad}^{2}\right)$ within $0.01 \%$ bandwidth. We have designed the general purpose beamline. This project allows one to assemble dedicated beamline (for, e.g., photolithography, EXAFS, x-ray material analysis, etc.). The radiation from both conventional magnet dipoles and wigglers as well would be utilized through this line. 
Fig. 5 depicts a sketch of the dipole vacuum chamber connected to the front-end for the case of channeling SR from a dipole magnet (a) and a wiggler (b). Choice of extracting angles is determined by the geometric consideration. The geometry chosen will provide up to 24 beamlines ( 21 of which are from dipole magnets and 3 are from insertion devices).

General layout of a SR line is shown in Fig. 6. The line comprises from: the front-end, the acoustic delay line, the mirrors chamber, the input part and the experimental station.
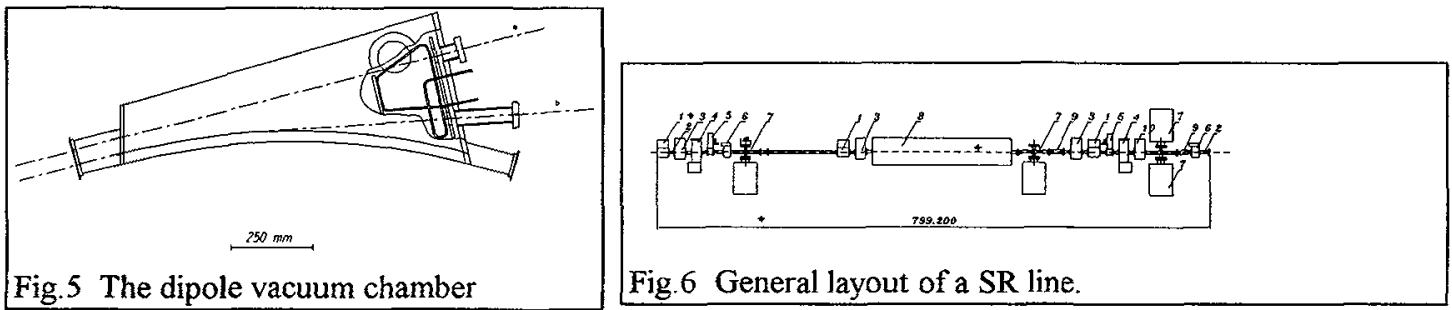

The beamline is supposed to operate in following modes:

1. X-ray lithography beamline (comprises from no optical elements inside). Transverse beam dimensions could be achieved within the range of $0-30 \mathrm{~mm}$.

2. The line with the turnable flat grid will be applied for experiments in physics and chemistry. This line comprised few optical elements covers photon frequency from VUV to the soft $x$-ray.

3 The toroidal grid monochromator line will be used in experiments requiring large photon flux and moderate resolution.

4. X-ray transmitting microscope.

5. The coronal angyography line.

\subsection{The Storage Ring Magnets [2]}

The dipole magnet will have a conventional $C$ type cross section. The rectangular magnet will be laminated, the laminates are $1.5 \mathrm{~mm}$ thick. The cross-section of the dipole magnet is seen in Fig. 7. The good-field region extends to $\pm 15 \mathrm{~mm}$ horizontally. The dipole parameters are given in Table 3 .

Table 3 Parameters of dipole magnet

Number of dipoles

12

Length, $\mathrm{m}$

Field strength $(\mathrm{E}=800 \mathrm{MeV}), \mathrm{T}$

Horizontal good field, $\mathrm{mm}$

Vertical good field, mm

Field index

3

Gap, mm 36

Pole width, mm 110

Number of turns in the winding 48

Cond cross section $(12.5 * 12.5 \varnothing 7.5), \mathrm{mm}^{2} \quad 99.5$

Exciting current range, $\mathrm{kA}$ $0.16 \div 1.1$

Magnet weight, kG 2000

24 quadrupole magnets are grouped in three families with 8 in each, the lenses in a family being connected in series. The poles of all the lenses are of one and the same profile The bore diameter is $50 \mathrm{~mm}$ and length is $20 \mathrm{~cm}$ for all quadrupole magnets. A cross section of the quadrupole magnet is shown in Fig. 8 . 


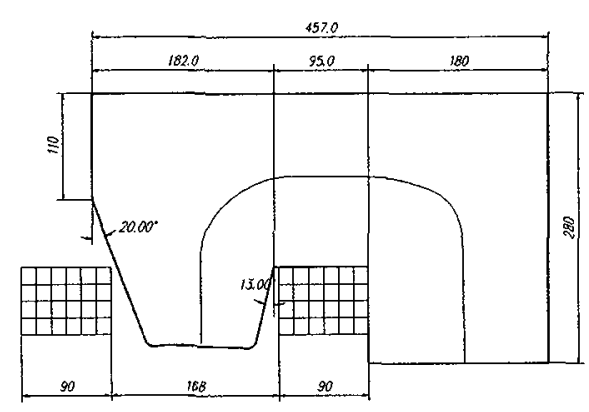

Fig. 7. Geometric dimensions of the ISI-800 dipole.

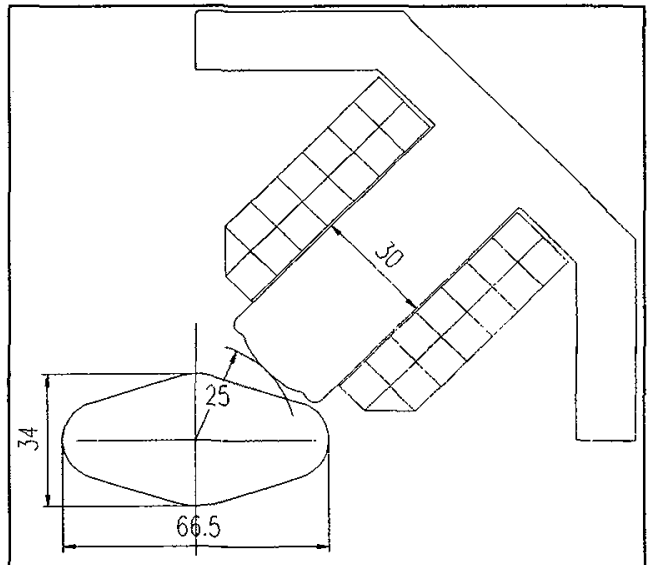

Fig. 9. Cross section quadrupole magnet.

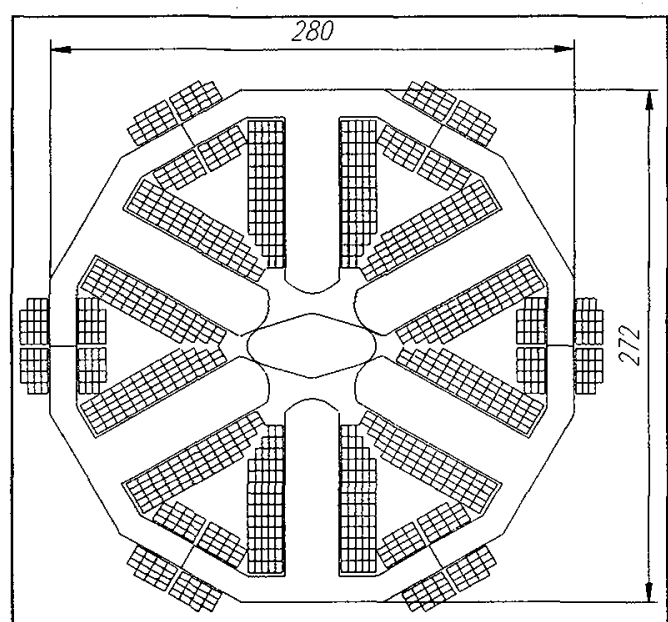

Fig. 9. Cross section the sextupole magnet. . is $0.03 \mathrm{~T}$ ).
The ISI-800 magnet lattice contains 4 families of sextupoles, total 24 magnets. To compensate the negative chromaticity $\xi_{x, z}$, which is equal to -7.14 , 7.36, four sextupole magnets are mounted at the curvilinear part of the trajectory of each superperiod and two sextupole magnets in the achromatic part of each superperiod correct the dynamic aperture. The aperture radius of sextupoles is $28 \mathrm{~mm}$, with a pole width of 28 $\mathrm{mm}$. The magnets are capable of producting a field of $200 \mathrm{~T} / \mathrm{m}^{2}$ over a length of $0.1 \mathrm{~m}$. The sextupole weight is $70 \mathrm{kG}$. The cross section of a sextupole is shown in Fig. 9. The parameters of the three different families are given in Table 4.

Table 4 Parameters of quadrupole magnet

Number of quadrupoles 24

Length, $\mathrm{m} \quad 0.2$

Bore length, $\mathrm{mm} \quad 50$

Gradient $(E=800 \mathrm{MeV}), \mathrm{T} / \mathrm{m} \quad 1.34$

Horizontal good field, mm $\quad 30$

Vertical good field, mm 20

Number of turns in the winding $\quad 48$

Copper cross section $(8 * 8 \varnothing 4.5), \quad 48$ $\mathrm{mm}^{2}$

Exciting current range, $\mathrm{kA}$

up 0.5

Quadrupole magnet weight, $\mathrm{kG}$

145

In addition to the main coils mounted on the poles, there are six back-leg coils which, given the correct excitation, can generate vertical and horizontal dipole fields with maximum corrector strength: vertical 3 $\mathrm{mTm}$, horizontal $3 \mathrm{mTm}$.

The synchrotron radiation facility ISI-800 will have a transfer line which has been designed for transporting $120 \mathrm{MeV}$ electrons from the linac to the storage ring. The transfer line includes 4 dipole magnets (rectangular, C-type, bend angle is 41.5 degrees, length is $29 \mathrm{~cm}$ ), 9 quadrupole magnets (length is $10 \mathrm{~cm}$, gradient is 15 $\mathrm{T} / \mathrm{m}$ ) and 4 bidirectional steering magnets.

Correction of the storage ring orbit is accomplished by 8 horizontal, 8 vertical sextupole steering elements (combined with the sextupoles) in the curvilinear part of the orbit and 8 bidirectional dipole steering magnets distributed in the straight sections (length is $10 \mathrm{~cm}$, field 


\subsection{RF-system}

Beam energy losses by synchrotron radiation and parasitic losses in the vacuum chamber walls are compensated with the help of a $10 \mathrm{~kW} \mathrm{RF}(699.3 \mathrm{MHz})$ system. The accelerating voltage of $200 \mathrm{kV}$ chosen in view of the Touschek lifetime, is provided by a single half-wave cavity, whose shape has been optimized against the shunt resistance at the main (operating) mode ( $\Omega$-cavity). As calculations and measurements indicate, this cavity has lower coupling impedances at higher-order modes than the cylindrical cavity has and, therefore, is less sensitive to the excitation of coupled oscillations of bunches.

\subsection{Vacuum}

Based on the reasonable 6-hour life of the zero-intensity beam we come to the conclusion that the residual gas pressure in the chamber should not exceed $5^{*} 10^{-9}$ Torr throughout the range of circulating currents at an operating energy of the beam. Pumping is produced by 40 sputtering $400 \mathrm{l} / \mathrm{s}$ pumps placed on the dipole magnet chambers and by the end of the defocusing quadrupole magnets.

Sixteen pickup stations and a current transformer are used to monitoring the beam in the storage ring.

\section{STATE AND PROGRESS OF THE ISI-800}

The design of the magnet, the vacuum, the RF and the indicating systems is completed. Also we have the project of the injector and the building.

Now we work on the modified TBA lattice, the beamlines, the design of the superconducting dipole magnet and the wiggler.

The injecting beam dynamics and process of increasing the beam energy up to the nominal one are under research. We also find the way to increase the operating energy up to $1 \mathrm{GeV}$.

\section{REFERENCES}

[1] Bar'yakhtar V. et al., "A Source of Synchrotron Radiation for Research and Technology Applications" PAC93, Washington, 1993; E. Bulyak et al, "Synchrotron radiation source", HEPACC-92, Hamburg, 1992.

[2] E.Bulyak, A.Gevchuk, P.Gladkikh, I.Karnaukhov, S.Kononenko, A.Mytsykov, A.Shcherbakov "The magnet system of the synchrotron radiation source ISI-800", MT13, Vancouver, 1993 